\title{
Forensic Feature-Comparison Expertise: Statistical Learning Facilitates Visual Comparison Performance
}

\author{
Bethany Growns \\ Arizona State University and University of New South Wales
}

\author{
Kristy A. Martire \\ University of New South Wales
}

\begin{abstract}
Forensic feature-comparison examiners in select disciplines are more accurate than novices when comparing visual evidence samples. This paper examines a key cognitive mechanism that may contribute to this superior visual comparison performance: the ability to learn how often stimuli occur in the environment (distributional statistical learning). We examined the relationship between distributional learning and visual comparison performance, and the impact of training about the diagnosticity of distributional information in visual comparison tasks. We compared performance between novices given no training (uninformed novices; $\mathrm{n}=32$ ), accurate training (informed novices; $\mathrm{n}=32$ ) or inaccurate training (misinformed novices; $\mathrm{n}=32$ ) in Experiment 1; and between forensic examiners $(\mathrm{n}=26)$, informed novices $(n=29)$ and uninformed novices $(n=27)$ in Experiment 2. Across both experiments, forensic examiners and novices performed significantly above chance in a visual comparison task where distributional learning was required for high performance. However, informed novices outperformed all participants and only their visual comparison performance was significantly associated with their distributional learning. It is likely that forensic examiners' expertise is domain-specific and doesn't generalise to novel visual comparison tasks. Nevertheless, diagnosticity training could be critical to the relationship between distributional learning and visual comparison performance.
\end{abstract}

Public Significance Statement: This study suggests that the ability to visually compare (or 'match') complex visual patterns can be improved by training individuals to use their knowledge of what features are rare or common in these patterns. This research has important practical implications for the performance of forensic feature-comparison examiners in the criminal justice system.

Data, analysis code, supplementary material: https://osf.io/bh3yg/

Keywords: expertise, forensic science, forensics, statistical learning, distributional learning

Forensic feature-comparison examiners determine if samples of unknown origin (e.g. fingerprints from a crime scene) originate from a known source (e.g. a suspect; Busey \& Parada, 2010; President's Council of Advisors on Science and Technology, 2016). These visual comparison tasks are challenging and involve a number of complex cognitive processes including perception, memory and decision-making (Busey \& Dror, 2011).

Bethany Growns, School of Social and Behavioural Sciences, Arizona State University; Kristy A. Martire, Department of Psychology, University of New South Wales.

This work was supported by an Australian Government Research Training Program (RTP) Scholarship to Bethany Growns and Australian Research Council Linkage Project Gran1 (LP160100008) to Kristy A. Martire.

Correspondence concerning this paper should be addressed to Bethany Growns, School of Social and Behavioural Sciences, Arizona State University - West Campus, 4701 W Thunderbird Rd, MC 3051, Glendale, AZ, 85069, USA, P: +1602-543-6031, E: bethany.growns@gmail.com
Yet, there is emerging evidence that examiners in some disciplines (e.g., fingerprints and handwriting) possess expertise in this task as they outperform novices (Bird, Found, Ballantyne, \& Rogers, 2010; Busey \& Vanderkolk, 2005; Kam, Fielding, \& Conn, 1997; Tangen, Thompson, \& McCarthy, 2011). Whilst the performance of examiners is not perfect and they do still make errors (see Thompson, Tangen, \& McCarthy, 2013; Ulery, Hicklin, Buscaglia, \& Roberts, 2012), examiners in select disciplines do outperform novices but we do not yet understand what underpins this expertise.

We are only just beginning to understand the perceptual and cognitive processes that may underlie forensic examiners' expertise. For example, fingerprint examiners process fingerprints differently to novices. Compared to lay people, fingerprint examiners show: holistic (Busey \& Vanderkolk, 2005; Gauthier \& Tarr, 1997; Gauthier, Williams, Tarr, \& Tanaka, 1998; Vogelsang, Palmeri, \& Busey, 2017; Yin, 1969) and nonanalytical processing (Thompson \& Tangen, 2014);

This paper is not the copy of record and may not exactly replicate the final, authoritative version of the article. 
less memory decay for fingerprints (Busey \& Vanderkolk, 2005; Thompson \& Tangen, 2014); more rapid identification of fingerprint target areas (Hicklin, Ulery, Busey, Roberts, \& Buscaglia, 2019); and more consistent eye fixation patterns to each other under time pressure (Busey et al., 2011). In this paper, we examine another cognitive process that may also contribute to the superior performance of forensic examiners: distributional statistical learning.

Information theory (Bruce \& Tsotsos, 2009; Shannon, 1948) suggests that stimuli distributions in the environment provide important diagnostic information in visual comparison tasks (Busey, Nikolov, Yu, Emerick, \& Vanderkolk, 2016). For example, consider a face comparison task where two images of faces are compared ('matched'). Suppose that both faces share two features: a common feature which many people in the population have (e.g. brunette hair); and a rare feature which relatively few people in the population have (e.g. a large scar). The distribution of those features occurring in the general population can assist when deciding whether the images are of the same person, or different people. A rare feature is more diagnostic for the purposes of identification because it is less frequent and shared by fewer people in the population (Bruce \& Tsotsos, 2009; Busey et al., 2016). Therefore, individuals who are better able to extract this distributional statistical information from their environment may be in a better position to excel in visual comparison tasks.

Statistical learning is theorised to be an innate but variable ability to extract statistical information from the environment across time and space (Siegelman, Bogaerts, Christiansen, \& Frost, 2017a; Siegelman, Bogaerts, \& Frost, 2017b; Siegelman \& Frost, 2015). Evidence shows that people can extract and encode the probability of objects co-occurring in time (transitional probabilities) and space (conditional probabilities) after only brief exposure and without instruction to do so (Fiser \& Aslin, 2001; Fiser \& Aslin, 2002; TurkBrowne, Jungé, \& Scholl, 2005). There is also evidence that people can learn distributions of stimuli occurring in the environment (known as distributional statistical learning; Growns, Siegelman, \& Martire, under review; Hasher \& Zacks, 1984; Maye, Werker, \& Gerken, 2002; Zacks \& Hasher, 2002).

Forensic examiners can learn distributions of stimuli occurring in forensic evidence - the very information that may contribute to their visual comparison performance. Martire, Growns, and Navarro (2018) investigated the distributional learning of novices and forensic document examiners who examine handwriting samples in forgery or fraud cases. Participants estimated the frequency of handwriting features from a database representative of US adult handwriting (Johnson, Vastrick, Boulanger, \& Schuetzner, 2017). The accuracy of these estimates was determined by calculating the difference between the estimated and 'groundtruth' frequencies. Document examiners from the US had significantly better estimation accuracy (i.e. lower absolute error at 20\%) than novices (26\%) and non-US document examiners (22\%). US examiners' mean estimates were also better calibrated and more precise. This indicates that the distributional learning of US document examiners is not only superior to novices but also to non-US document examiners - who have less experience with US handwriting.

Critically, distributional information has also been shown to increase forensic examiners' visual comparison performance. Busey et al. (2016) demonstrated that fingerprint examiners' comparison accuracy is better for rare, high diagnostic regions of fingerprints, than common, lower diagnostic regions. Busey et al. (2016) utilised the Attention via Information Maximisation model (AIM; Bruce \& Tsotsos, 2009; Busey et al., 2016) to identify high and low diagnostic features and regions in a set of fingerprints. Fingerprint examiners' subsequent visual comparison accuracy was significantly more accurate for partial prints containing rare, high diagnostic 'AIM' regions than prints containing less diagnostic 'AIM' regions. This demonstrates that fingerprint examiners' accuracy is better when they have access to perceptual information that is rare and diagnostic.

However, examiners' accuracy was only improved in this study using computationally-derived distributional information that was directly provided to them. They were not required to acquire or derive diagnostic information themselves. Given examiners likely extract distributional information via exposure to forensic evidence, it is not known whether they could rely on their own incidental distributional learning to facilitate visual comparison performance. It is also unclear whether they could rely on solely on their incidental learning, or whether they would also need some explicit understanding about the relationship between diagnosticity and distributional information (as per information theory).

Forensic examiners have some explicit understanding of what information is diagnostic in visual comparison tasks. Towler, White, and Kemp (2017) asked forensic facial examiners and novices to provide similarity ratings between facial features (e.g. ears) whilst 
completing a face comparison task. Facial examiners were not only outperformed novices overall, but their facial feature ratings were significantly more diagnostic of identity than novices' ratings. Importantly, they also reported that diagnostic features were more useful to their decisions than novices. This demonstrates that examiners were not only able to identify which features were rare and diagnostic to improve their visual comparison performance, but also demonstrated explicit knowledge of the usefulness of these diagnostic features. Thus, both access to distributional information and an explicit understanding of the diagnosticity of distributional information may be required for superior visual comparison performance.

In this paper we report two experiments examining: 1) whether incidental distributional statistical learning contributes to visual comparison performance; and 2) whether explicit knowledge about diagnosticity (as per information theory) affects this relationship. In Experiment 1 , we compare distributional learning and visual comparison performance of novices given no training or training in the use of diagnostic information in visual comparison tasks (either accurate or inaccurate). In Experiment 2 , we compare distributional learning and visual comparison performance between forensic examiners, and novices given no training or accurate training. In both experiments, we control for any pre-existing differences in incidental distributional learning by using novel visual stimuli.

\section{Experiment 1}

Theoretically-informed training modules have been shown to increase novice accuracy in domain-specific tasks ranging from radiology to chicken sexing (Biederman \& Shiffrar, 1987; Chen, HolcDorf, McCusker, Gaillard, \& Howe, 2017; Evered, Walker, Watt, \& Perham, 2014; Hornsby \& Love, 2014; Roads, $\mathrm{Xu}$, Robinson, \& Tanaka, 2018). Experiment 1 examined the distributional learning and visual comparison performance of novices given different types of diagnosticity training. If incidental distributional learning contributes to visual comparison performance, we predict that novices will perform significantly above chance in both distributional learning and visual comparison tasks, and a significant positive association between performance on both tasks. If explicit knowledge about diagnosticity effects this relationship, we would expect: significantly better visual comparison performance in novices given accurate diagnosticity training (informed novices), compared to those given no training (uninformed novices) or inaccurate diagnosticity training (misinformed novices); and a stronger association between informed novices' distributional learning and visual comparison performance, compared to uninformed or misinformed novices.

\section{Method}

\section{Design}

Experiment 1 was a 3-factor between-subjects design where participants were randomly assigned to receive no diagnosticity training (uninformed novices), accurate diagnosticity training (informed novices), or inaccurate diagnosticity training (misinformed novices). All participants completed an exposure phase where they viewed pattern exemplars, a test phase where they completed distributional learning measures and then a visual comparison phase. Data, analysis scripts and supplementary materials for Experiment 1 are available at https://osf.io/bh3yg/. This study was not pre-registered.

\section{Participants}

Participants were 96 undergraduate students from a large university in eastern Australia (uninformed novices: $=\mathrm{n}=32$; informed novices: $\mathrm{n}=32$; misinformed novices: $\mathrm{n}=32$ ) who were 19.29 years of age on average $(\mathrm{SD}=1.68$, range $=17-26)$. The majority of the sample was male (53.1\%). Sample size was determined by the number of participants available during the university semester. Recruitment ceased at the end of semester.

\section{Materials}

Apparatus. All displays were presented on a 24-inch BENQ LCD monitor attached to a HP Workstation Z220, running Windows 7 SP1.

Stimuli. During the exposure phase, participants viewed 60 complex visual patterns manipulated to contain features in a pseudo-randomised order (i.e. appeared in the same random order generated when programming the experiment) to minimise error variance across participants (Mollon, Bosten, Peterzell, \& Webster, 2017).

Exemplars were created by adding three features to the base pattern in opposing pairs (see Figure 1 and left panel of Figure 2). Within the set of 60 exemplars, feature probability was varied so that features co-occurred with three joint probabilities (JP). Feature pairs were either: rare with a JP of 0.1 , uncommon $(\mathrm{JP}=0.3)$, or 


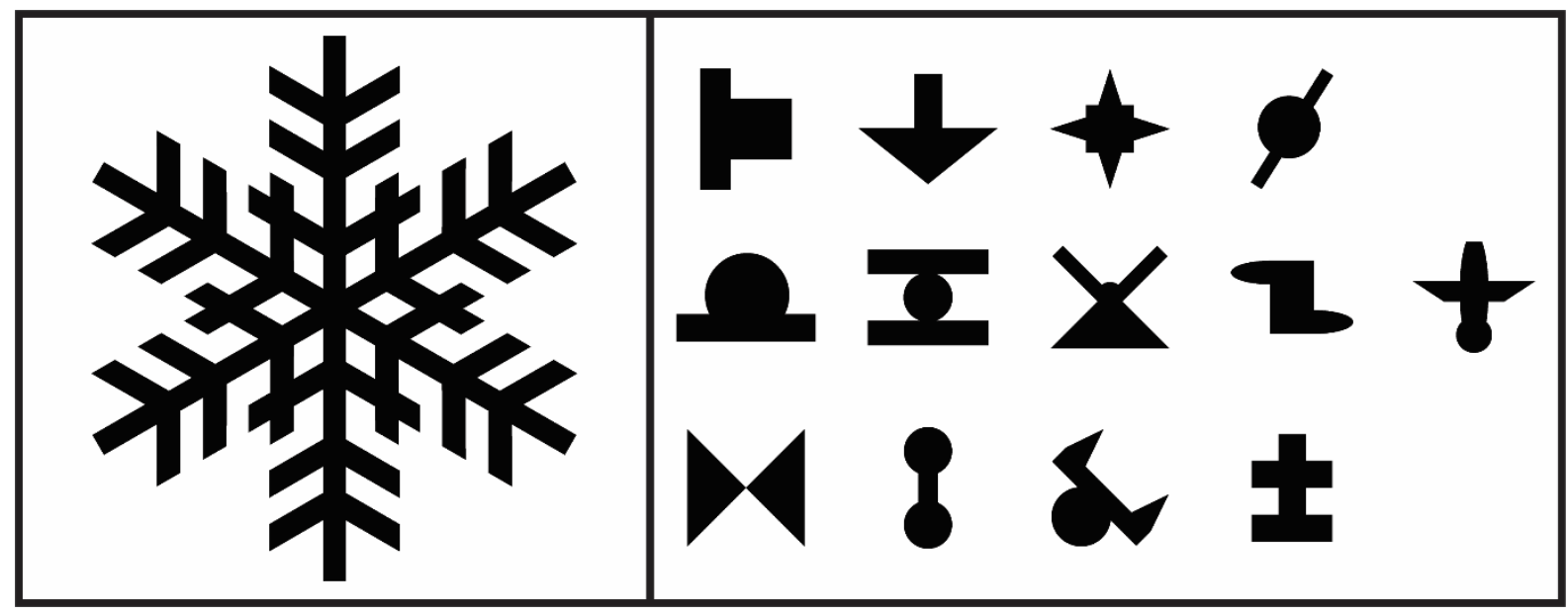

Figure 1. Base pattern (left panel) and features (right panel) used to create experimental stimuli

common $(\mathrm{JP}=0.7)$. See Growns, Siegelman \& Martire (under review) and supplementary materials for a detailed description of exposure phase stimuli.

Attention-check task. Participants completed a 1back N-back cover task during the exposure phase to ensure they attended to the exemplars. Participants were asked to press the spacebar when they saw the same feature in the same location on consecutive exemplars. Participants were required to correctly complete a practice N-back trial before beginning the exposure phase.

Visual comparison task. Participants completed 360 visual comparison trials in a pseudo-randomised order. On each comparison trial, participants viewed one clear exemplar (i.e. the same as an exposure phase exemplar; left panel in Figure 2) and one blurred exemplar with two visible features (created by applying the Gaussian blur filter at the 25-pixel level in Adobe Photoshop to ensure obscured features were unidentifiable; right panel in Figure 2; see Pilot 1 in supplementary materials). Participants were asked: 'How likely is it that these patterns are the same?' and were required to press a number between ' 1 ' (Very Unlikely) to '6' (Very Likely).

On each trial, the visible features in the blurred exemplars were also present in the same location as the clear exemplar (see Figure 2). Consequently, participants had to determine whether the two exemplars matched given that they had two features in common. The location of the visible features was pseudo-randomised on each trial. Before completing the visual comparison task, participants viewed GIFs demonstrating how the blur was applied to exemplars, revealing the same or different exemplars underneath.
Participants completed three different visual comparison trial types, where visible features had a rare, uncommon or common joint probability as described above. Participants completed more common $(n=84)$ than uncommon $(n=36)$ trials, and more uncommon than rare ( $n=12$ per rare feature) trials. Given that statistical learning is a fluid process and can change over time (Siegelman, Bogaerts, Kronenfeld, \& Frost, 2018), this ensured that the relative joint probabilities from the exposure phase were maintained so that any change in participants' distributional learning between the test and visual comparison phases would be minimal.

Trials were either match (where exemplars were identical beneath the blur) or a non-match trials (where exemplars differed beneath the blur). The ratio of match to non-match trials in rare, uncommon and common trials was designed to accurately reflect the relationship between probability and diagnosticity as per information theory. Specifically, there were less common match trials than rare match trials, as frequently occurring features are less indicative of a match.

The ratio of match to non-match trials within each joint probability reflected the random probability that two exemplars would 'match' from the sub-population of exemplars sharing the same visible features (with replacement). See Table S-1 in supplementary materials for detailed information about the visual comparison task trials.

Diagnosticity training. Informed and misinformed novices completed a training module prior to the visual comparison task, whilst uninformed novices proceeded directly to the visual comparison task. Informed novices were told that rare features were more diagnostic 

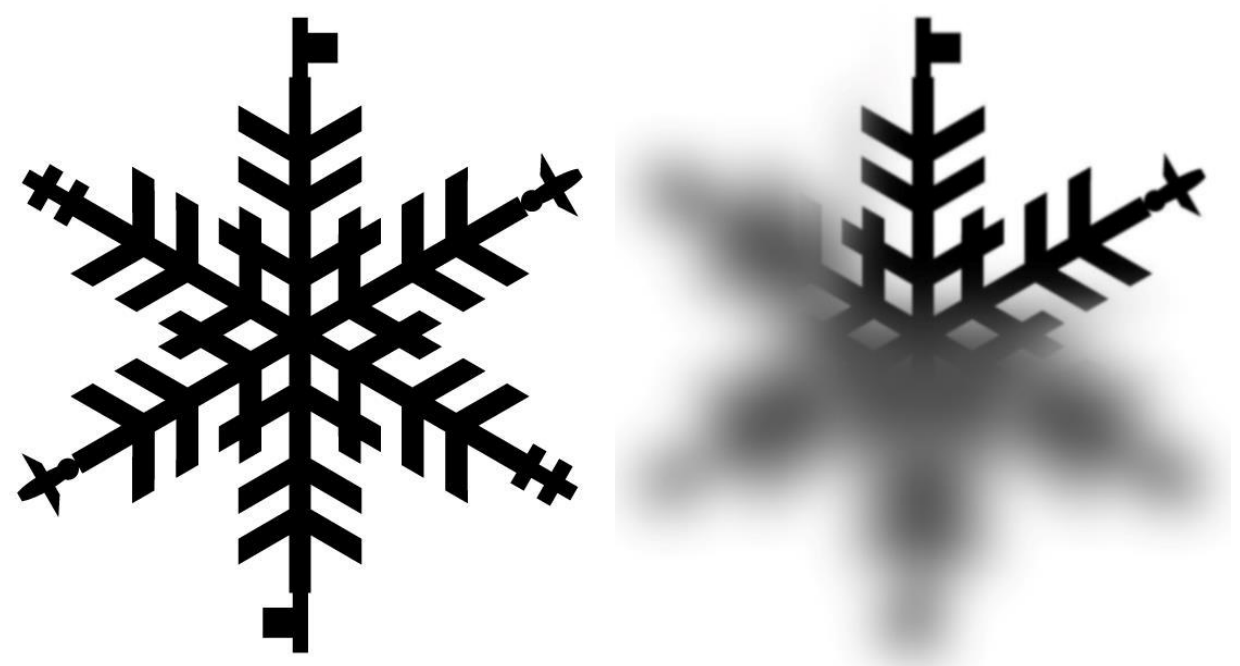

Figure 2. Example of clear and blurred exemplars used in Experiments 1 and 2

than common features, while uninformed novices were told that common features were more diagnostic than rare features.

Informed novices read a scenario where they were asked to imagine they were a police officer needing to compare photos of people. They were told they could use their knowledge about how rare or common features were when deciding if the photos were of the same or different people. They were then presented with two hypothetical cases: one where two photos shared a rare feature (i.e. a large scar; Case 1); and one where two photos shared a common feature (i.e. brunette hair; Case 2).

They were asked to imagine that they knew only one photo pair was of the same person (the other photo pair was of different people) and were asked choose which case it would be (Case 1 or 2). Participants were then provided with feedback. Those that chose Case 1 received a 'Correct!' message, and those that chose Case 2 received an 'Incorrect!' message.

They then completed six trials where they were asked to imagine they needed to complete visual comparison tasks for exemplars sharing rare or common features (i.e. fingerprints or shoeprints), receiving corrective feedback on each trial.

Misinformed novices were erroneously told common features were more diagnostic than rare features. They completed the same police and additional scenarios as informed novices but received the opposite feedback (i.e. 'Correct!' in common cases, and 'Incorrect!' for rare cases). See supplementary materials for verbatim training modules.

\section{Dependent Measures}

\section{Distributional Learning}

Participants completed two measures of distributional learning: discrimination judgements and frequency estimates.

Discrimination judgements. Participants completed 33 pattern recognition trials and 12 pattern completion trials in a pseudo-randomised order (45 trials in total). During pattern recognition trials participants were asked to choose which feature or pairs of features were more familiar from an array including two, three or four features or pairs of features. During pattern completion trials, participants were asked to choose the feature "that best completes the pair" from an array of two or three features. The correct answers were determined based on the true joint probabilities from the exposure phase. Participants were instructed to ignore feature orientation and location when making their choices. Group-level chance performance was calculated by aggregating the different probabilities of responses for each trial (Siegelman et al., 2017b). In this case, chance performance was accuracy on 20.5 trials (45.5\%).

Frequency estimates. Each of the 13 different features in the exemplar set had a specific frequency of occurrence resulting in the JPs as described above (either $0.1,0.3,0.7$ or 1.0$)$. Participants were presented each feature in a pseudo-randomised order and were asked 'what proportion of the time did this shape occur in the images that you saw?' Absolute error was calculated for each participant by subtracting the true feature 
frequency from the estimated feature frequency for each feature, then averaging across all 13 individual estimates. Lower absolute error values indicate better estimation accuracy.

\section{Visual Comparison Accuracy}

Visual comparison accuracy was measured using d' and C (Phillips, Saks, \& Peterson, 2001; Stanislaw \& Todorov, 1999). d' measures sensitivity to the presence of a target signal in stimuli. Higher scores indicate better sensitivity. C measures response bias. A tendency to say 'match' results in a negative bias score, while the tendency to say 'non-match' results in positive bias scores.

\section{Procedure}

Participants were randomly assigned to training condition. All participants provided demographic information and then completed the 8-minute exposure phase while completing the N-back task. The exposure phase comprised two blocks of 60 exemplars, each displayed for 3-seconds each, with an interstimulus interval of 1-second. Participants then completed the distributional learning test phase by providing discrimination judgements and frequency estimates. Next, participants in the accurate and inaccurate training conditions completed the relevant training module, before all participants completed the visual comparison task. Upon completion of the experiment, participants were thanked and debriefed and misinformed novices received verbal corrective feedback about diagnosticity.

\section{Results}

\section{Distributional Learning}

One-sample t-tests revealed that discrimination judgements were significantly better than chance in all training conditions (i.e. 13.25 trials; see Table 1), and descriptive statistics for absolute error can be seen in Figure 3. Discrimination judgements and absolute error were analysed using ANOVAs with training as the between-subjects factor (see Figure 3). Neither discrimination judgements $\left(F_{(2,93)}=1.30, p=.277, \eta_{\mathrm{p}} 2=.03\right)$, nor absolute error $\left(F_{(2,93)}=.21, p=.810, \eta_{\mathrm{p}} 2=.01\right)$ significantly differed by training condition.

\section{Visual Comparison Accuracy}

One-sample t-tests revealed that sensitivity was significantly above chance for uninformed and informed novices (i.e. 0; see Table 1), but not misinformed novices. Bias was below zero in all conditions which indicates a preference to say 'match' over 'non-match.' Between-groups ANOVAs were conducted to compare sensitivity and bias by training condition (see Figure 4). Bias did not significantly differ by condition $\left(F_{(2,93)}=\right.$ $\left..38, p=.686, \eta_{\mathrm{p}} 2=.01\right)$, whereas sensitivity $\operatorname{did}\left(F_{(2,93)}\right.$ $\left.=5.54, p=.005, \eta_{\mathrm{p}} 2=.11\right)$.

Follow-up comparisons revealed that informed novices' sensitivity was significantly higher than uninformed novices' $(t(62)=2.52, p=.014,95 \% \mathrm{CI}=.15$ $1.29)$ and misinformed novices $(t(62)=2.88, p=.005$,

Table 1

Experiment 1: One-sample t-test results by group for discrimination judgements and sensitivity

\begin{tabular}{lccccc}
\hline & Chance & Mean (SD) & $d f$ & $t$ & $p$ \\
\hline \multicolumn{5}{c}{ Discrimination Judgements } \\
\hline Uninformed Novices & 20.5 & $40.90(7.26)$ & 31 & 15.48 & $<.001$ \\
Informed Novices & 20.5 & $40.66(7.62)$ & 31 & 12.03 & $<.001$ \\
Misinformed Novices & 20.5 & $39.00(8.01)$ & 31 & 11.35 & $<.001$ \\
& \multicolumn{7}{c}{ Sensitivity (d') } & & \\
& 0 & $.41(.89)$ & 31 & 2.59 & .014 \\
Uninformed Novices & 0 & $1.13(1.35)$ & 31 & 4.71 & $<.001$ \\
Informed Novices & 0 & $.18(1.26)$ & 31 & .82 & .416 \\
Misinformed Novices & 0 & & & & \\
\hline
\end{tabular}



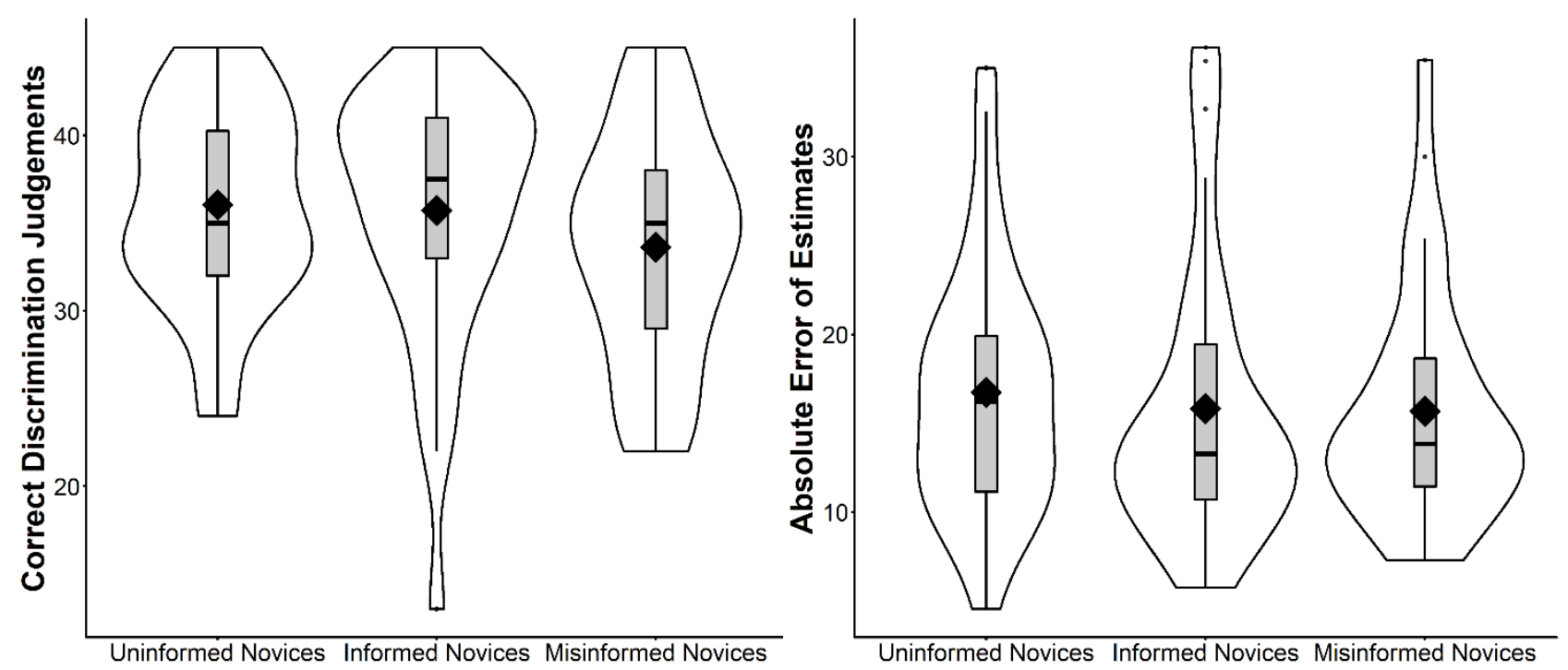

Figure 3. Experiment 1: Discrimination judgement accuracy (left panel) and absolute error (right panel) by condition

Note: width of violin plots corresponds to frequency of scores; and the box-and-whisker plots represent the median (the middle black line), mean (diamond), first and third quartiles (boxes either side of the line) and no further than $1.5 \mathrm{x}$ the interquartile range (the 'whiskers'; Wickham, 2016)

95\% CI $=.29-1.59)$. No significant differences were found between uninformed and misinformed novices $(t(62)=.82, p=.417,95 \% \mathrm{CI}=-.32-.76)$.

\section{Distributional Learning and Visual Comparison Performance}

We examined the association between distributional learning and visual comparison performance with regression models using the base stats package in $\mathrm{R}$ (see Figure 5). Each model predicted visual comparison performance (sensitivity or bias) from distributional learning measures (discrimination judgements or absolute error) with training, and the interaction between training and each distributional learning measure. This allowed us to examine if distributional learning predicted visual comparison performance in a way that differed between groups. Thus, we report the interactions in-text and all other regression output can be found in the supplementary materials.

\section{Discrimination Judgements}

Sensitivity was significantly predicted by discrimination judgements, group and their interaction $\left(F_{(5,90)}\right.$ $=4.28, p=.002$, adj. $\left.\mathrm{R}^{2}=.15\right)$. The interaction be- tween informed and misinformed novices was significant $\left(\beta=.12, t_{(90)}=2.78, p=.007\right)$. As informed novices' discrimination accuracy increased, sensitivity significantly also increased, compared to misinformed novices' accuracy increasing as sensitivity decreased. The interaction between uninformed and informed novices was not significant $\left(\beta=.05, t_{(90)}=1.18, p=\right.$ $.241)$, nor was the interaction between uninformed novices and misinformed novices $\left(\beta=-.06, t_{(90)}=\right.$ $1.33, p=.188)$.

Bias was not significantly predicted by discrimination judgements, group or their interaction $\left(F_{(5,90)}=\right.$ $1.02, p=.414$, adj. $\left.\mathrm{R}^{2}=.00\right)$. See Table $\mathrm{S}-3$ for the full regression output of both discrimination judgement models.

\section{Absolute Error}

Sensitivity was significantly predicted by absolute error, group and their interaction $\left(F_{(5,90)}=2.89, p=\right.$ .018 , adj. $\mathrm{R}^{2}=.09$ ). However, none of the pairwise interactions significantly contributed to the model. Bias was not significantly predicted by absolute, group and their interaction $\left(F_{(5,90)}=.79, p=.558\right.$, adj. $\left.\mathrm{R}^{2}=-.01\right)$. See Table S-4 for the full regression output of both absolute error models. 

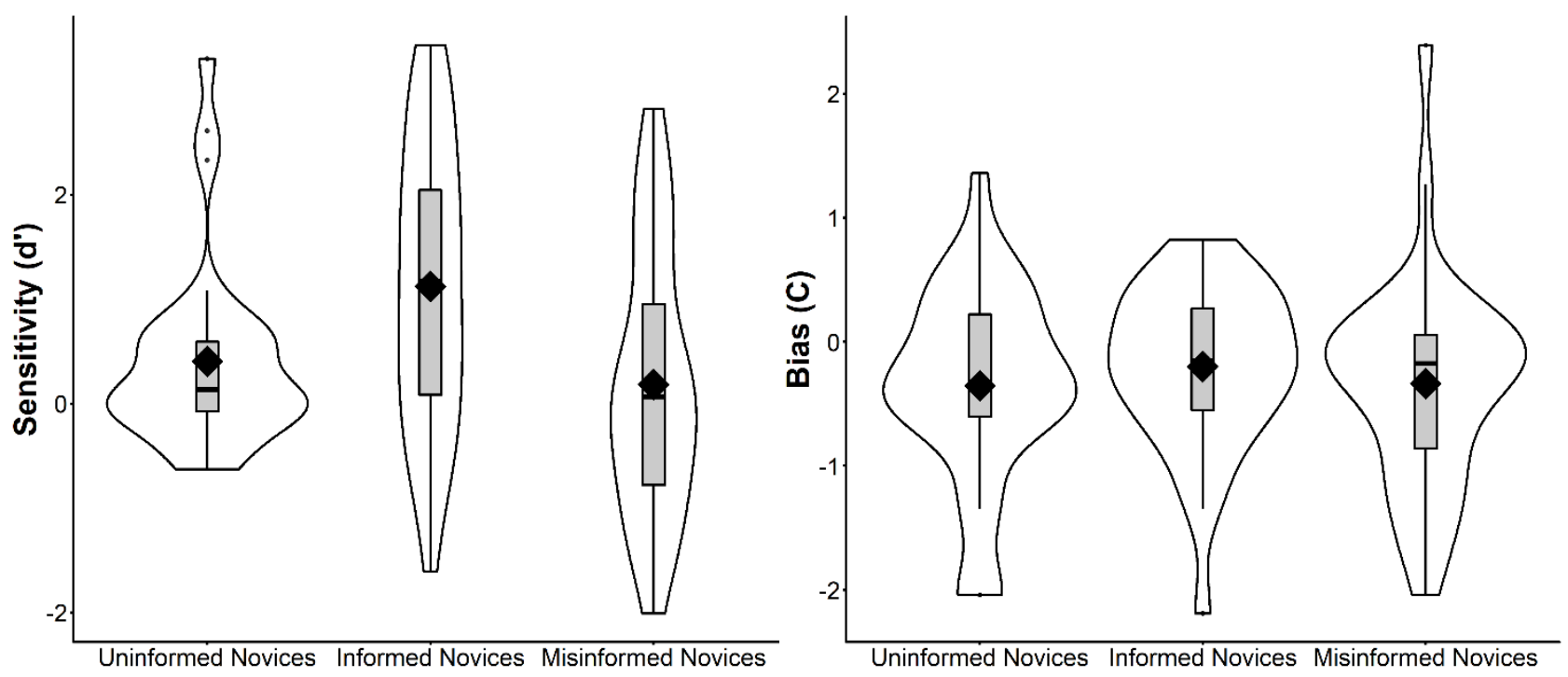

Figure 4. Experiment 1: Sensitivity (left panel) and bias (right panel) by group

Note: width of violin plots corresponds to frequency of scores; and the box-and-whisker plots represent the median (the middle black line), mean (diamond), first and third quartiles (boxes either side of the line) and no further than $1.5 \mathrm{x}$ the interquartile range

\section{Discussion}

Experiment 1 examined the distributional learning and visual comparison performance of novices given different types of diagnosticity training. Consistent with past research (Fiser \& Aslin, 2001; Growns et al., under review; Zacks \& Hasher, 2002), participants were able to learn how often features occurred in exemplars after only brief exposure: discrimination judgements were significantly above chance and absolute error was low, and did not differ by training. Visual comparison performance (sensitivity) was also significantly better than chance on a task where high performance could only be achieved via distributional learning. Just as computationally-derived distributional information facilitated fingerprint examiners' visual comparison performance (Busey et al., 2016), Experiment 1 demonstrated that incidental distributional learning can also contribute to visual comparison accuracy. However, performance and its association with distributional learning varied by diagnosticity training.

Whilst both uninformed and informed novices' visual comparison performance was significantly above chance, informed novices were significantly outperformed uninformed and misinformed novices. Moreover, only informed novices' distributional learning and visual comparison performance were reliably positively associated, compared to misinformed novices. These effects were not driven by any changes in participants' response bias as bias (C) did not significantly differ between groups, and bias was not significantly associated with distributional learning. This suggests that whilst there is some naïve ability in applying distributional learning in visual comparison tasks (as observed in uninformed novices), explicit training about the diagnosticity of distributional information improves this ability. It is also critical to the relationship between distributional learning and visual comparison performance. We further explore this relationship in Experiment 2.

Overall, Experiment 1 demonstrated that uninformed and informed novices were able to use their incidental distributional learning to facilitate visual comparison performance, regardless of training. However, accurate diagnosticity training improves this ability, and inaccurate training impairs it. Diagnosticity training is also critical to the underlying association between distributional learning and visual comparison performance. Thus, forensic examiners' superior visual comparison performance may be driven both by their incidental distributional learning and explicit knowledge about diagnosticity. In Experiment 2, we examined this by comparing distributional learning and 

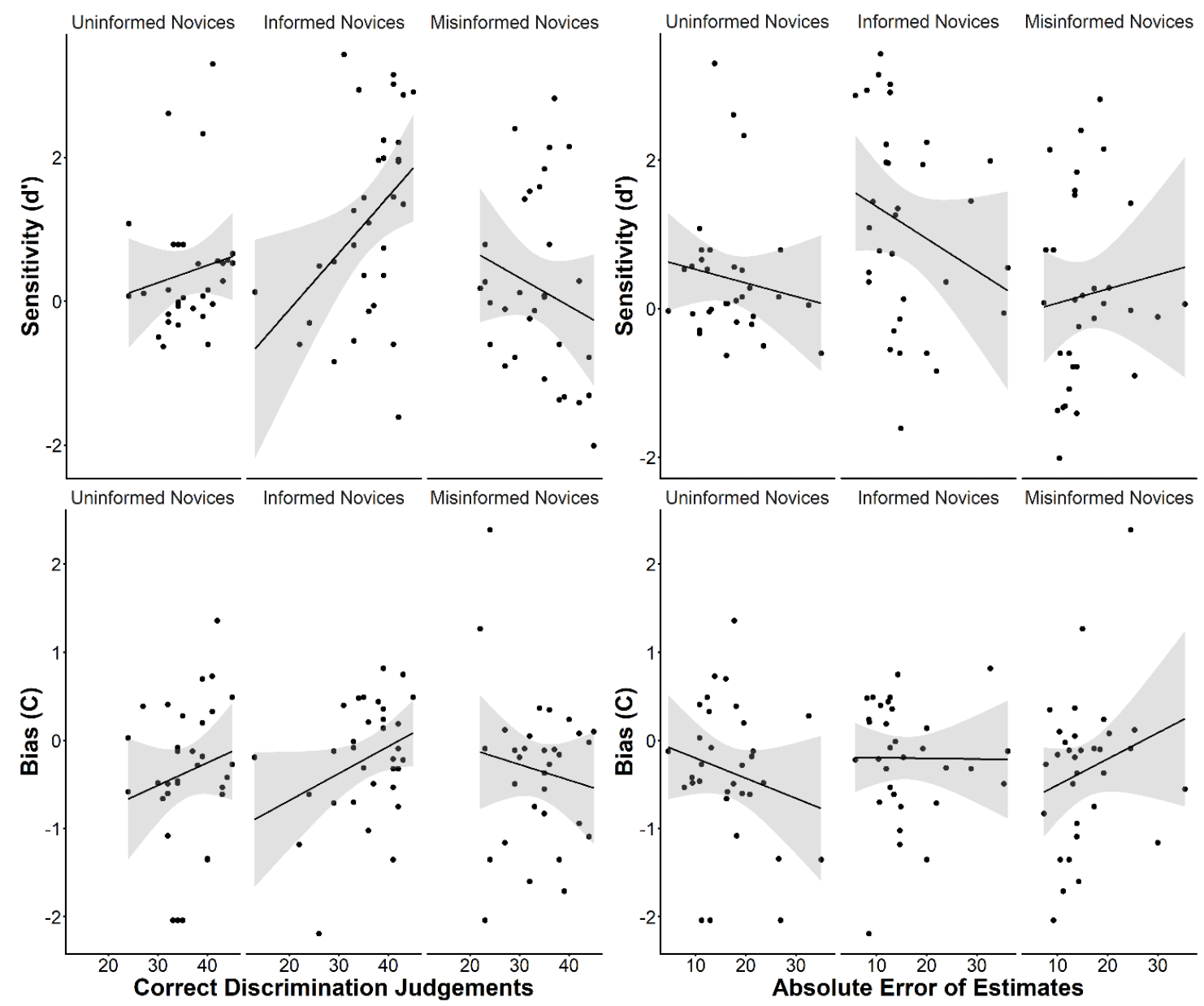

Figure 5. Experiment 1: Visual comparison performance (sensitivity: top panels; bias: bottom panels) and distributional learning measures (discrimination judgements: left panels; absolute error: right panels) by group. Linear regression lines are displayed with $95 \%$ confidence interval bands (Wickham, 2016)

visual comparison performance between forensic examiners and informed and uninformed novices.

\section{Experiment 2}

Forensic examiners have better explicit understanding of what features are diagnostic in forensic comparison tasks than novices (Towler et al., 2017). Yet it is not clear whether they are able to identify diagnostic features on some perceptual level, or whether this knowledge translates to a broader domain-general understanding of the relationship between diagnosticity and frequency. It is possible that forensic examiners develop this understanding and can then generalise this concept to performance in another task. Experiment 2 investigates this hypothesis.
If examiners have a domain-general understanding of the relationship between diagnosticity and frequency, we would expect examiners to perform similar to informed novices in terms of average visual comparison performance and the association between distributional learning and visual comparison performance. We would also expect their performance to differ to uninformed novices.

Conversely, if examiners do not have this domaingeneral understanding, we would expect their performance to be similar to uninformed novices. 


\section{Method}

\section{Design}

Experiment 2 was a quasi-experimental 3-factor between-subjects design where participants were either forensic examiners, or novices randomly assigned to receive no training or accurate training as per Experiment 1. Experiment 2 was pre-registered at https://aspredicted.org/sa5yx.pdf, and the data, analysis scripts and supplementary material are available at https://osf.io/bh3yg/.

\section{Participants}

Recruitment procedure. We recruited forensic examiners through a snowball sampling method via emails sent to forensic laboratories, mailing lists and universities. From the total snowball sample $(n=225)$, 38 individuals reported that they were a forensic feature-comparison examiner or forensic scientist. All other participants from the snowball sample were excluded $(\mathrm{n}=188)$, including a heterogenous group of other self-reported perceptual 'experts' (e.g. birdwatchers; Shen, Mack, \& Palmeri, 2014) that we originally pre-registered our intention to recruit to compare with forensic examiners. Sample size was determined by the number of participants recruited during our preregistered time period.

We then recruited an equivalent number of novices via Amazon Mechanical Turk (MTURK) who were randomly assigned to receive no training $(n=36)$ or accurate training $(n=36)$. Participants from MTURK received \$3USD to participate in the 30-minute experiment. A $\$ 100$ VISA gift-card was offered to the participant with the most accurate visual comparison performance.

Inclusion criteria. All participants were required to complete a CAPTCHA before beginning the experiment (Von Ahn, Maurer, McMillen, Abraham, \& Blum, 2008), and all MTURK participants were required to: reside in the United States, have greater than 99\% approval for the MTURK assignments and to have completed more than 100 MTURK tasks.

Exclusion criteria. Participants were excluded if they did not pass two data quality criteria: if they did not correctly answer $3 / 5(60 \%)$ of the attention-check questions or failed a question to identify bots (i.e., 'Please enter the 8th word in the sentence below'; $\mathrm{n}=$ $24)$; or if they participated in our pilot studies $(n=4)$. As only $45.5 \%(n=50)$ of the original sample met the pre-registered inclusion criteria $(4 / 5 ; 80 \%)$, we lowered the inclusion threshold $(3 / 5 ; 60 \%)$ to better represent the whole sample and report those results here. Results using the pre-registered inclusion criteria are reported in supplementary materials but were not meaningfully different.

Final participant groups. The final sample comprised 26 forensic feature-comparison examiners, 27 uninformed novices, and 29 informed novices. Novices were 32.75 years of age on average $(\mathrm{SD}=10.36$, range $=18-61)$, half the sample was male $(50 \%$; female $=$ $48.2 \%$; transgender $=1.8 \%$ ) and all spoke English as their first language $(100 \%)$. Forensic examiners were 40.96 years of age on average $(\mathrm{SD}=10.00$, range $=18$ $59)$, the majority of the sample was female $(53.8 \%)$ and the majority spoke English as their first language (76.9\%). Examiners had 13.04 years' experience $(\mathrm{SD}=$ 9.10 , range $=1-30$ ) on average in their primary area of specialisation (i.e., questioned documents: $26.9 \%$; firearms: $23.1 \%$; image comparison: $23.1 \%$; fingerprints: 11.5\%; footwear: $7.7 \%$; gunshot residue and drugs: $7.6 \%)$. Examiners reported their primary employers were government institutions $(23 \%)$, or police $(61.5 \%)$ or private laboratories $(15.4 \%)$.

\section{Materials}

To encourage the participation of professionals (Wray \& Gates, 1996), we reduced the length of all phases in the study compared to Experiment 1 and removed the N-back task from the exposure phase (due to high attrition in a pilot experiment; see Pilot 2 in supplementary materials). We pre-tested these amendments and they did not affect distributional learning or visual comparison performance (see Pilot 3 in supplementary materials).

Experimental platform. Participants completed the experiment online using Qualtrics (2005). Participants were instructed to adjust the zoom settings on their browser (if necessary) to ensure that all images were able to be seen.

Exposure phase stimuli. During the exposure phase, participants completed one block of 60 exemplars from Experiment 1 in a pseudo-random order. Each exemplar was viewed for 3-seconds with an ISI of 1-second.

Visual comparison task. Participants completed a 90-trial version of the visual comparison task in a pseudo-randomised order. As in Experiment 1, the task was composed of rare, common or uncommon joint probability trials. Relative base rates and ratio of match-nonmatch trials was maintained from the exposure phase (see Table S-2 in supplementary materials). Trials were randomly selected from rare, uncommon 

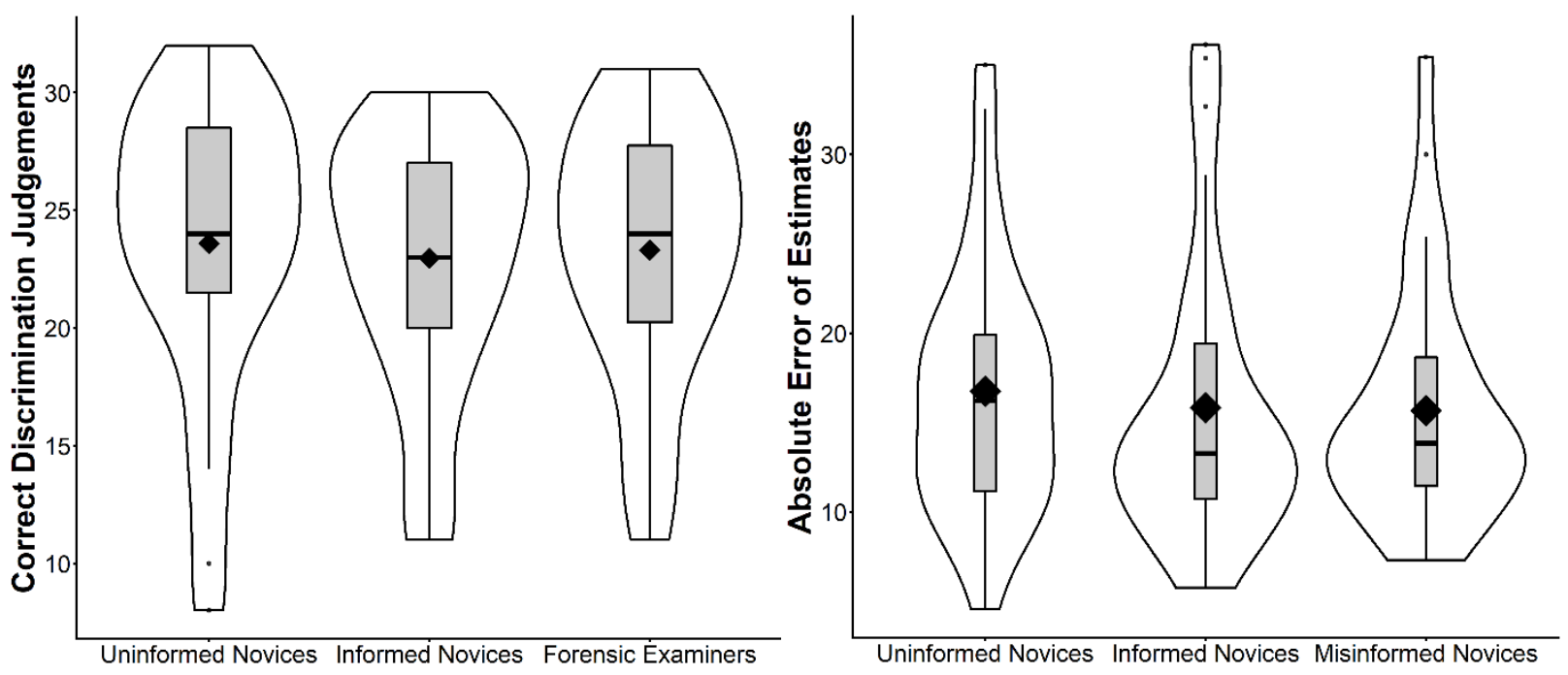

Figure 6. Experiment 2: Discrimination judgement accuracy (left panel) and absolute error (right panel) between training groups

Note: width of violin plots corresponds to frequency of scores; and the box-and-whisker plots represent the median (the middle black line), mean (diamond), first and third quartiles (boxes either side of the line) and no further than $1.5 \mathrm{x}$ the interquartile range (the 'whiskers')

and common trials used in Experiment 1 whilst maintaining the relative base rates.

Training module. Prior to the visual comparison task, informed novices completed the same accurate diagnosticity training module from Experiment 1.

\section{Dependent Measures}

Discrimination judgements. Participants completed 21 pattern recognition trials and 12 pattern completion trials with accuracy determined by the base rates from the exposure phase. Chance performance for the discrimination judgement task in Experiment 2 was accuracy on 13.25 trials $(40.2 \%)$.

Frequency estimates. Participants were shown 13 features in a pseudo-randomised order and asked to estimate their frequency as in Experiment 1.

\section{Procedure}

Forensic examiners were recruited first using a snowball sampling method. Next, an equivalent number of novices were recruited via MTURK. Novices were randomly assigned to a training condition. All participants provided demographic information first, then completed the exposure phase, test phase and comparison phases. Informed novices completed the accurate diagnosticity training module before the comparison phase, while examiners and uninformed novices did not. Upon completion of the experiment, participants viewed the debrief screen and were thanked for their participation.

\section{Results}

\section{Distributional Learning}

One-sample t-tests revealed that discrimination judgements were significantly better than chance in all training groups (see Table 2). Discrimination judgements and absolute error were analysed using ANOVAs with training group as the between-subjects factors (see Figure 6). Neither discrimination judgements $\left(F_{(2,79)}=.09, p=.918, \eta_{\mathrm{p}} 2<.01\right)$ nor absolute error $\left(F_{(2,79)}=.35, p=.703, \eta_{\mathrm{p}} 2=.01\right)$ significantly differed between groups.

\section{Visual Comparison Accuracy}

One-sample t-tests revealed that sensitivity was significantly above chance in all training groups (see 
Table 1

Experiment 1: One-sample t-test results by group for discrimination judgements and sensitivity

\begin{tabular}{|c|c|c|c|c|c|}
\hline & Chance & Mean (SD) & $d f$ & $t$ & $p$ \\
\hline \multicolumn{6}{|c|}{ Discrimination Judgements } \\
\hline Uninformed Novices & 20.5 & $40.90(7.26)$ & 31 & 15.48 & $<.001$ \\
\hline Informed Novices & 20.5 & $40.66(7.62)$ & 31 & 12.03 & $<.001$ \\
\hline Misinformed Novices & 20.5 & $39.00(8.01)$ & 31 & 11.35 & $<.001$ \\
\hline \multicolumn{6}{|c|}{ Sensitivity (d') } \\
\hline Uninformed Novices & 0 & $.41(.89)$ & 31 & 2.59 & .014 \\
\hline Informed Novices & 0 & $1.13(1.35)$ & 31 & 4.71 & $<.001$ \\
\hline Misinformed Novices & 0 & $.18(1.26)$ & 31 & .82 & .416 \\
\hline
\end{tabular}

Table 2). Bias was below zero in all conditions which indicates a preference to say 'match' over 'non-match.' Sensitivity and bias were analysed using ANOVAs with group as the between-subjects factor (see Figure 7). Bias did not significantly differ by group $\left(F_{(2,79)}=\right.$ $\left..71, p=.495, \eta_{\mathrm{p}} 2=.03\right)$, whereas sensitivity did $\left(F_{(2,79)}\right.$ $=5.28, p=.007, \eta_{\mathrm{p}} 2=.12$ ).

Follow-up comparisons revealed that sensitivity was significantly higher for informed than uninformed novices $\left(t_{(54)}=2.29, p=.026,95 \% \mathrm{CI}=.07-1.04\right)$ and forensic examiners $\left(t_{(53)}=2.95, p=.005,95 \% \mathrm{CI}=.23\right.$ -
1.12). No significant differences were found between uninformed novices and examiners $\left(t_{(51)}=2.72, p=\right.$ $.472,95 \% \mathrm{CI}=-.26-.57)$.

\section{Distributional Learning and Visual Comparison Performance}

We examined the association between distributional learning and visual comparison performance with regression models as in Experiment 1 (see Figure 8). We
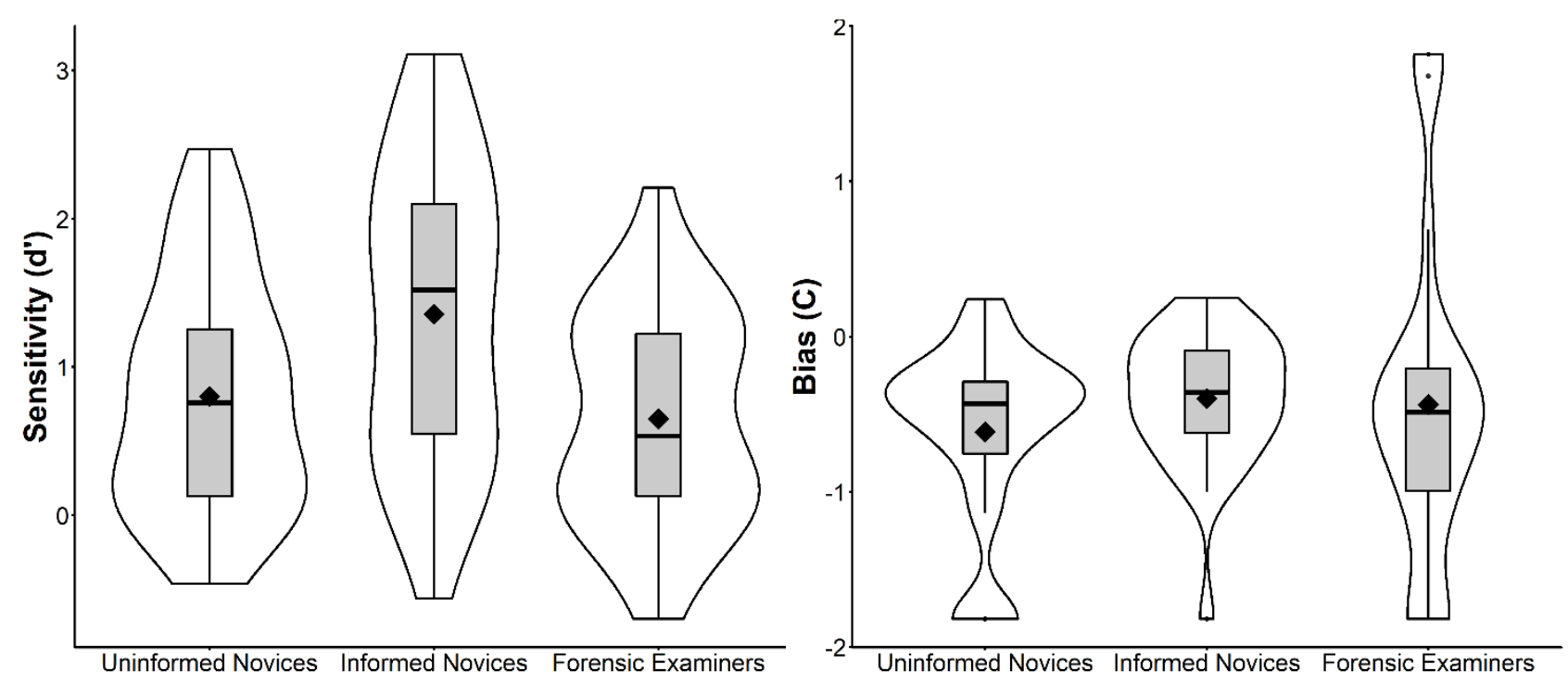

Figure 7. Experiment 2: Sensitivity (left panel) and bias (right panel) between groups

Note: width of violin plots corresponds to frequency of scores; and the box-and-whisker plots represent the median (the middle black line), mean (diamond), first and third quartiles (boxes either side of the line) and no further than $1.5 \mathrm{x}$ the interquartile range (the 'whiskers') 

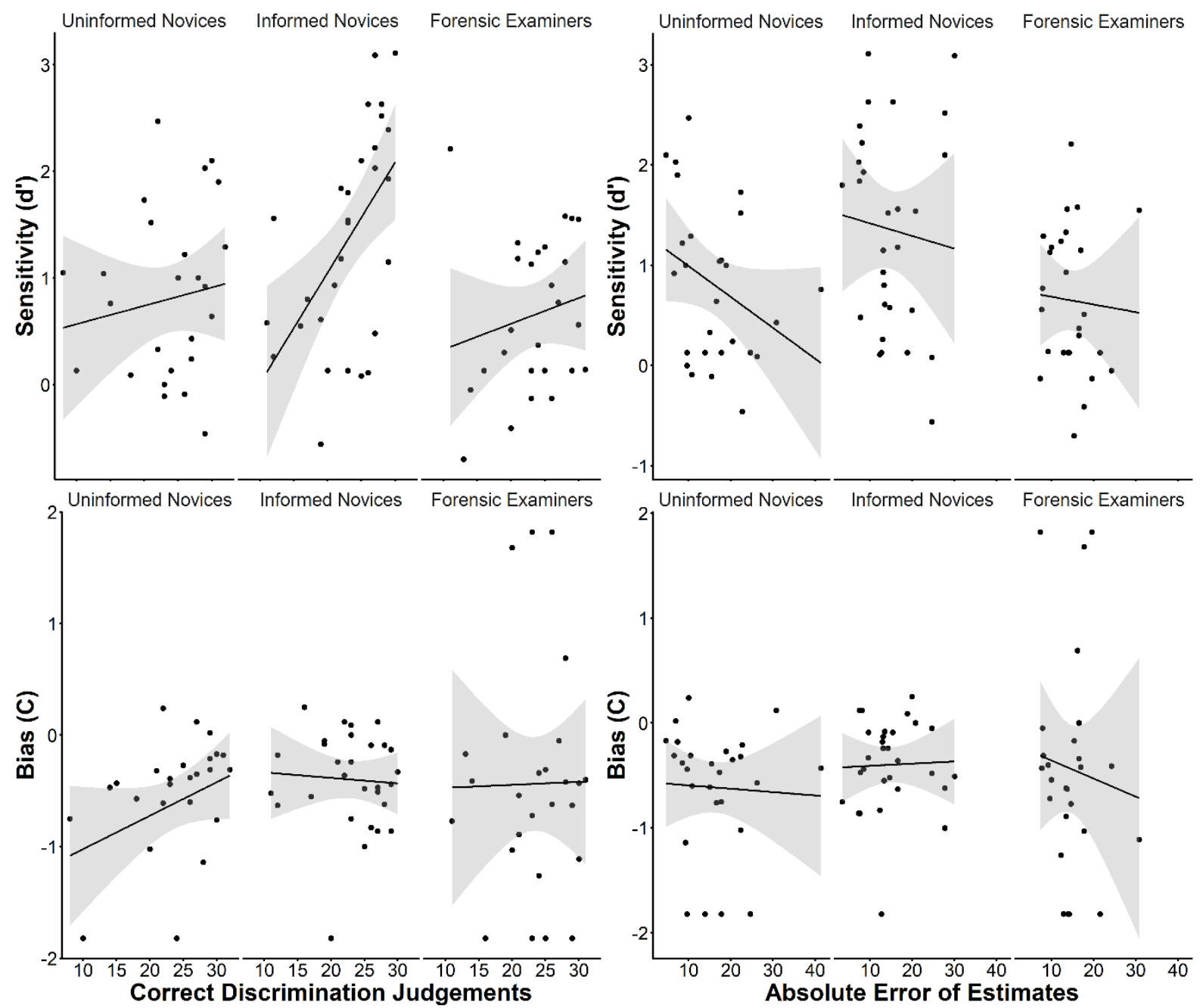

Figure 8. Experiment 2: Sensitivity (top panels) and bias (bottom panels) and distributional learning measures (discrimination judgements: left panels, and absolute error: right panels) between groups. Linear regression lines are displayed with 95\% confidence interval bands

report the interactions between condition and each distributional learning measure in-text and all other regression output can be found in the supplementary materials.

\section{Discrimination Judgements}

Sensitivity was significantly predicted by discrimination judgements, group and their interaction $\left(F_{(5,76)}=\right.$ $5.41, p<.001$, adj. $\mathrm{R} 2=.21)$. The interaction between uninformed and informed novices was significant $(\beta=$ $\left..09, t_{(76)}=2.30, p=.024\right)$.

As informed novices' discrimination accuracy increased, sensitivity significantly also increased, compared to misinformed novices' accuracy and sensitivity. The interaction between uninformed and examiners was not significant $\left(\beta=.01, t_{(76)}=.18, p=.857\right)$, nor was the interaction between informed novices and examiners $\left(\beta=.08, t_{(76)}=1.96, p=.053\right)$.

Bias was not significantly predicted by discrimination judgements, group or their interaction $\left(F_{(5,76)}=.64\right.$, $p=.673$, adj. $\mathrm{R} 2=-.02$ ). See Table S-5 for the full regression output of both discrimination judgement models.

\section{Absolute Error}

Sensitivity was significantly predicted by absolute error, group and their interaction $\left(F_{(5,76)}=2.66, p=.029\right.$, adj. R2 = .09). However, none of the pairwise interactions significantly contributed to the interaction. Bias was not significantly predicted by absolute, group and their interaction $\left(F_{(5,76)}=.37, p=.869\right.$, adj. $\left.\mathrm{R} 2=-.04\right)$. See Table S-6 for the full regression output of both absolute error models. 


\section{Discussion}

Experiment 2 examined the association between distributional learning and visual comparison performance between forensic examiners and novices given no training or accurate diagnosticity training. Consistent with Experiment 1, distributional learning occurred and was equivalent between groups. Visual comparison performance was also significantly above chance in all groups. However, informed novices significantly outperformed both examiners and uninformed novices in the visual comparison task. Further, only informed novices' distributional learning was again reliably and positively associated with visual comparison performance, compared to uninformed novices. Neither of these results was attributable to differences in bias. Experiment 2 further supports the inference that there is a naïve ability to apply distributional learning to visual comparison tasks, but that knowledge of the relationship between diagnosticity and distributional information improves that ability.

However, it is unclear whether explicit knowledge about diagnosticity contributes to the domain-specific superior visual comparison performance of forensic examiners. Whilst forensic examiners' visual comparison performance was significantly above chance (in a task where success relies on distributional learning), it did not significantly differ from uninformed novices. And the association between forensic examiners' distributional learning and visual comparison performance also did not significantly differ from uninformed novices. Whilst the association between discrimination judgements and visual comparison performance also didn't significantly differ between forensic examiners and informed novices, these associations are likely not reliably similar $(\mathrm{p}=.053)$. The small number of participants recruited in the eligible time pre-registered time period may have impacted our ability to detect significant differences between these associations (further discussed below). Overall, examiners may benefit from the same naïve ability as novices to apply distributional learning in visual comparison tasks, they may not have generalisable explicit knowledge about the diagnosticity of distributional information in visual comparison tasls.

\section{General Discussion}

This paper sought to better understand the expertise of forensic feature-comparison examiners by investigating whether incidental distributional learning facilitates visual comparison performance. We also examined whether explicit knowledge about diagnosticity and distributional information (as per information theory) contributes to this relationship. Overall, we found clear evidence that distributional learning contributes to visual comparison performance. We also found evidence of an association between distributional learning and visual comparison accuracy that relied on explicit diagnosticity training.

Consistent with previous research (Fiser \& Aslin, 2001; Zacks \& Hasher, 2002), individuals were able to extract and encode distributional information from brief exposure to novel stimuli. Participants' discrimination judgements were significantly above chance and absolute error was relatively low in both experiments $(\mathrm{M}=22.15$ and $\mathrm{M}=15.24$ in Exps. 1 and 2 respectively). This paper also demonstrates that individuals can apply this distributional learning to facilitate accuracy in another domain: visual comparison performance. In a visual comparison task that relied distributional learning to achieve high accuracy, participants' performance was significantly above chance across both experiments (except misinformed novices in Exp. 1). Just as computationally-derived distributional information improves forensic examiners' visual comparison accuracy (Busey et al., 2016), we identified the first evidence that incidental distributional learning can also facilitate visual comparison performance.

Forensic examiners' explicit knowledge of diagnostic features improves their visual comparison performance (Towler et al., 2017). This paper also demonstrated that explicit knowledge about distributional information and diagnosticity improves visual comparison performance. Whilst uninformed novices' visual comparison performance hints at a naïve ability to apply distributional learning in visual comparison tasks, diagnosticity training significantly improved this ability. Although statistical learning is theorised to be both partially implicit and explicit in nature (Arciuli, von Koss Torkildsen, Stevens, \& Simpson, 2014; Batterink, Reber, Neville, \& Paller, 2015), explicit knowledge improves the application of statistical learning in visual comparison tasks.

This explicit knowledge also strengthens the relationship between distributional learning and visual comparison performance. Informed novices' distributional learning was significantly associated with visual comparison performance across two experiments (compared to misinformed novices in Experiment 1 and uninformed novices in Experiment 2). This suggests that diagnosticity training helped better distributional learners to utilise their learning to improve their visual comparison performance. Our results suggest that both incidental distributional learning and explicit 
knowledge about diagnosticity (as per information theory) are implicated in superior visual comparison performance.

However, forensic examiners' visual comparison performance was equivalent to uninformed novices, and examiners' distributional learning was not associated with visual comparison performance (compared to uninformed novices). This suggests that examiners do not have a superior ability to implicitly apply distributional learning in visual comparison tasks with novel stimuli. They also do not appear to have generalised knowledge about distributional information and diagnosticity that allows better distributional learners to improve their visual comparison performance.

On one hand, this could suggest that neither incidental distributional learning, nor explicit knowledge of diagnosticity, play a role in the superior visual comparison performance of forensic examiners (Busey \& Vanderkolk, 2005; Kam et al., 1997; Tangen et al., 2011). Yet, superior domain-specific distributional learning has been observed among forensic examiners (Martire et al., 2018), and their visual comparison performance is improved with both computationally-derived distributional informational (Busey et al., 2016) and explicit knowledge of diagnostic features (Towler et al., 2017). However, these results were obtained within examiners' areas of professional practice. Thus, it is more likely that forensic examiners' superior distributional learning and explicit diagnosticity knowledge are restricted to their domain of expertise, and do not generalise to novel, unfamiliar stimuli. Expertise is often shown to be narrow and domain-specific (Bedard \& Chi, 1992; Chase \& Simon, 1973) sometimes even stimulus-specific (Martire et al., 2018). Thus, examiners' superior visual comparison performance may only be facilitated by domain-specific distributional learning or explicit diagnosticity knowledge (e.g. pertaining specifically to faces or fingerprints).

Indeed, informed novices were effectively acting as 'experts' with our novel stimuli. They were able to draw upon their distributional learning of the novel stimuli, and were given diagnosticity training that it could be directly applied to the visual comparison task at hand. As a result, informed novices performed significantly better than forensic examiners and uninformed novices. In contrast, forensic examiners could not rely on their domain-specific superior distributional learning to improve visual comparison performance in this experimental context. It is also likely that their knowledge about diagnosticity was domain-specific and not easily generalisable to unfamiliar comparison tasks. Consequently, the forensic examiners performed equivalent to uninformed novices who had access to the same resources.

Future research needs to explore these possibilities within forensic examiners domains of practice. However, it must be noted that it will be practically challenging to control for prior exposure to distributional information in a meaningful way (e.g., based on number of comparisons, number of cases, years of practice etc.). Future research should also explore whether explicit diagnosticity training can further improve forensic examiners' knowledge of domain-specific diagnostic features. Given emerging calls for forensic science training to include human factors research (Earwaker, Nakhaeizadeh, Smit, \& Morgan, 2019), it is possible that a brief training intervention could be used to improve examiners' real-world visual comparison performance.

\section{Practical Implications}

Despite the firm theoretical basis for the importance of distributional learning in visual comparison performance, its utility may be hindered by variability in perceptual and cognitive processes between and within examiners. For example, fingerprint examiners differ in the number of minutiae that they identify in the same fingerprint - and the same examiner even identifies different numbers of minutiae in the same print at two different timepoints (Dror et al., 2011). Fingerprint examiners' visual comparison decisions are also influenced by contextual information - they decide not to conduct comparisons due to 'insufficient' visual information in more cases with lower-profile crimes (e.g. vehicle theft) than higher-profile crimes (e.g. murder; Earwaker, Morgan, Harris, \& Hall, 2015). These examples illustrate the variety of external factors that can influence what examiners perceive and process in forensic evidence. Consequently, examiners may not always 'see' the same objective visual information in front of them. Even in a perfect world - where examiners learn feature frequencies and utilise this knowledge in comparison tasks, their ability to do so may be impacted by how they process stimuli. This highlights the importance of further research into the perceptual and cognitive processes surrounding forensic expertise.

This paper also focused solely on the role distributional learning in visual comparison performance - potentially discounting the important role that other forms of statistical learning may play. We took this approach because distributional learning is an elementary statis- 
tical property relevant to visual comparison performance (Bruce \& Tsotsos, 2009; Busey et al., 2016). However, this focus discounts the potentially important role of spatial learning in visual comparison performance. For example, minutiae in fingerprints have distributional frequencies but also appear in unique spatial relationships that would increase their diagnosticity (Phillips et al., 2001). Further, minutiae can be more or less common in certain regions of fingerprints (Busey \& Parada, 2010). Statistical learning research has demonstrated that people also learn spatial co-occurrences (Fiser \& Aslin, 2001; Fiser \& Aslin, 2005). Thus, it is likely that forensic examiners learn and use relevant diagnostic spatial statistics in visual-comparison tasks. Future research should explore this possibility.

\section{Limitations and Future Directions}

Although we identified a significant association between distributional learning and visual comparison performance across both experiments, it is important to note this relationship was somewhat inconsistent. Informed novices' visual comparison performance was significantly associated with discrimination judgement accuracy in both experiments, but only in comparison to misinformed novices in Experiment 1 and uninformed novices in Experiment 2. Our sample sizes were restricted by specific time periods and difficulty recruiting professional participants (Wray \& Gates, 1996). This may have impacted our ability to find significant differences between associations in groups. Nevertheless, a reliable and positive association was seen between informed novices' distributional learning and visual comparison accuracy across two experiments. Future research should continue to investigate this relationship using larger sample sizes recruited over longer time periods.

It is also notable that absolute error was not significantly associated with visual comparison performance across both experiments. This may be because recognising features with different frequencies (i.e. discrimination judgements) and explicitly recalling feature frequency (i.e. frequency estimates) are two distinct aspects of distributional learning. Given that human memory is typically theorised to contain separate, but related, recognition and recall systems (Haist, Shimamura, \& Squire, 1992), distributional learning may also rely on different memory processes. The ability to recognise feature frequencies may be a form of distributional learning that is associated with visual comparison performance, whereas the ability to recall these features may not be. However, evidence also suggests that absolute error is a less reliable measure of distributional learning than discrimination judgement accuracy (Growns, Siegelman \& Martire, under review). It may therefore also have poorer predictive performance. Indeed, people are generally poor at explicitly estimating probabilities and their estimates tend to be miscalibrated with true probabilities (Brenner, Koehler, Liberman, \& Tversky, 1996; Lee \& Danileiko, 2014; Martire et al., 2018; Zhang \& Maloney, 2012). Thus, we cannot draw strong conclusions about the relationship between estimation accuracy and visual comparison performance. Future research should continue to explore this relationship.

\section{Conclusion}

Overall, we have demonstrated that distributional learning contributes to visual comparison performance. We have also identified a significant association between distributional learning and visual comparison performance, but only for those given accurate diagnosticity training. Our results suggest that both distributional learning and knowledge of the relationship between diagnosticity and distributional information are both important for expert visual comparison performance. However, further research is required to assess the generalisability of these findings to real word forensic feature-comparison expertise.

\section{References}

Arciuli, J., von Koss Torkildsen, J., Stevens, D. J., \& Simpson, I. C. (2014). Statistical learning under incidental versus intentional conditions. Frontiers in Psychology, 5, 1-8.

Batterink, L. J., Reber, P. J., Neville, H. J., \& Paller, K. A. (2015). Implicit and explicit contributions to statistical learning. Journal of Memory and Language, 83, 62-78.

Bedard, J., \& Chi, M. T. (1992). Expertise. Current Directions In Psychological Science, 1(4), 135-139.

Biederman, I., \& Shiffrar, M. M. (1987). Sexing day-old chicks: A case study and expert systems analysis of a difficult perceptual-learning task. Journal of Experimental Psychology: Learning, Memory, and Cognition, 13(4), 640-645.

Bird, C., Found, B., Ballantyne, K., \& Rogers, D. (2010). Forensic handwriting examiners' opinions on the process of production of disguised and simulated signatures. Forensic Science International, 195(1-3), 103-107.

Brenner, L. A., Koehler, D. J., Liberman, V., \& Tversky, A. (1996). Overconfidence in probability and frequency judgments: A critical examination. Organizational Behavior and Human Decision Processes, 65(3), 212-219.

Bruce, N. D., \& Tsotsos, J. K. (2009). Saliency, attention, and visual search: An information theoretic approach. Journal of Vision, 9(3), 5-5. 
Busey, T., Nikolov, D., Yu, C., Emerick, B., \& Vanderkolk, J. (2016). Characterizing human expertise using computational metrics of feature diagnosticity in a pattern matching task. Cognitive Science, 41, 1717-1759.

Busey, T., Yu, C., Wyatte, D., Vanderkolk, J., Parada, F., \& Akavipat, R. (2011). Consistency and variability among latent print examiners as revealed by eye tracking methodologies. Journal of Forensic Identification, 61(1), 60-91.

Busey, T. A., \& Dror, I. E. (2011). Special abilities and vulnerabilities in forensic expertise. In A. McRoberts (Ed.), The Fingerprint Sourcebook (pp. 1-23). Washington, DC, USA: U.S. Department of Justice, National Institute of Justice.

Busey, T. A., \& Parada, F. J. (2010). The nature of expertise in fingerprint examiners. Psychonomic Bulletin \& Review, 17(2), $155-160$.

Busey, T. A., \& Vanderkolk, J. R. (2005). Behavioral and electrophysiological evidence for configural processing in fingerprint experts. Vision Research, 45(4), 431-448.

Chase, W. G., \& Simon, H. A. (1973). Perception in chess. Cognitive Psychology, 4(1), 55-81.

Chen, W., HolcDorf, D., McCusker, M. W., Gaillard, F., \& Howe, P. D. (2017). Perceptual training to improve hip fracture identification in conventional radiographs. PLoS ONE, $12,1-11$.

Dror, I. E., Champod, C., Langenburg, G., Charlton, D., Hunt, H., \& Rosenthal, R. (2011). Cognitive issues in fingerprint analysis: Inter-and intra-expert consistency and the effect of a 'target'comparison. Forensic Science International, 208(1-3), 10-17.

Earwaker, H., Morgan, R. M., Harris, A. J., \& Hall, L. J. (2015). Fingermark submission decision-making within a UK fingerprint laboratory: Do experts get the marks that they need? Science \& Justice, 55(4), 239-247.

Earwaker, H., Nakhaeizadeh, S., Smit, N. M., \& Morgan, R. M. (2019). A cultural change to enable improved decision-making in forensic science: A six phased approach. Science \& Justice, $1-11$.

Evered, A., Walker, D., Watt, A. A., \& Perham, N. (2014). Untutored discrimination training on paired cell images influences visual learning in cytopathology. Cancer Cytopathology, 122(3), 200-210.

Fiser, J., \& Aslin, R. N. (2001). Unsupervised statistical learning of higher-order spatial structures from visual scenes. Psychological Science, 12(6), 499-504.

Fiser, J., \& Aslin, R. N. (2002). Statistical learning of higherorder temporal structure from visual shape sequences. Journal of Experimental Psychology: Learning, Memory, \& Cognition, 28(3), 458-467.

Fiser, J., \& Aslin, R. N. (2005). Encoding multielement scenes: statistical learning of visual feature hierarchies. Journal of Experimental Psychology: General, 134(4), 521.

Gauthier, I., \& Tarr, M. J. (1997). Becoming a 'Greeble' expert: Exploring mechanisms for face recognition. Vision Research, 37(12), 1673-1682.

Gauthier, I., Williams, P., Tarr, M. J., \& Tanaka, J. (1998). Training 'Greeble' experts: a framework for studying expert object recognition processes. Vision Research, 38(15-16), 2401-2428.

Growns, B., Siegelman, N., \& Martire, K. A. (under review). The multi-faceted nature of visual statistical learning: individual differences in learning conditional and distributional regularities across time and space. Psychological Bulletin \& Review.
Haist, F., Shimamura, A. P., \& Squire, L. R. (1992). On the relationship between recall and recognition memory. Journal of Experimental Psychology: Learning, Memory, and Cognition, 18(4), 691.

Hasher, L., \& Zacks, R. T. (1984). Automatic processing of fundamental information: The case of frequency of occurrence. American Psychologist, 39(12), 1372-1388.

Hicklin, R. A., Ulery, B. T., Busey, T. A., Roberts, M. A., \& Buscaglia, J. (2019). Gaze behavior and cognitive states during fingerprint target group localization. Cognitive Research: Principles and Implications, 4(1), 12.

Hornsby, A. N., \& Love, B. C. (2014). Improved classification of mammograms following idealized training. Journal of Applied Research in Memory and Cognition, 3(2), 72-76.

Johnson, M. E., Vastrick, T. W., Boulanger, M., \& Schuetzner, E. (2017). Measuring the frequency occurrence of handwriting and handprinting characteristics. Journal of Forensic Sciences, 62(1), 142-163.

Kam, M., Fielding, G., \& Conn, R. (1997). Writer identification by professional document examiners. Journal of Forensic Sciences, 42(5), 778-786.

Lee, M. D., \& Danileiko, I. (2014). Using cognitive models to combine probability estimates. Judgment and Decision Making, 9(3), 259-273.

Martire, K. A., Growns, B., \& Navarro, D. J. (2018). What do the experts know? Calibration, precision, and the wisdom of crowds among forensic handwriting experts. Psychonomic Bulletin \& Review, 25(6), 2346-2355.

Maye, J., Werker, J. F., \& Gerken, L. (2002). Infant sensitivity to distributional information can affect phonetic discrimination. Cognition, 82(3), 102-111.

Mollon, J. D., Bosten, J. M., Peterzell, D. H., \& Webster, M. A. (2017). Individual differences in visual science: What can be learned and what is good experimental practice? Vision Research, 141, 4-15.

Phillips, V. L., Saks, M. J., \& Peterson, J. L. (2001). The application of signal detection theory to decision-making in forensic science. Journal of Forensic Sciences, 46(2), 294-308.

President's Council of Advisors on Science and Technology. (2016). Forensic Science in Criminal Courts: Ensuring Scientific Validity of Feature-Comparison Methods. United States: Executive Office of the President's Council of Advisors on Science and Technology

Roads, B. D., Xu, B., Robinson, J. K., \& Tanaka, J. W. (2018). The easy-to-hard training advantage with real-world medical images. Cognitive Research: Principles and Implications, $3(1), 38$.

Shannon, C. E. (1948). A Mathematical Theory of Communication. Bell System Technical Journal, 27(3), 379423.

Shen, J., Mack, M. L., \& Palmeri, T. J. (2014). Studying realworld perceptual expertise. Frontiers in Psychology, 5, 1-6.

Siegelman, N., Bogaerts, L., Christiansen, M. H., \& Frost, R. (2017a). Towards a theory of individual differences in statistical learning. Philosophical Transactions of the Royal Society B: Biological Sciences, 372, 1-10.

Siegelman, N., Bogaerts, L., \& Frost, R. (2017b). Measuring individual differences in statistical learning: Current pitfalls and possible solutions. Behavior Research Methods, 49(2), 418-432.

Siegelman, N., Bogaerts, L., Kronenfeld, O., \& Frost, R. (2018). Redefining "learning" in statistical learning: what does an online measure reveal about the assimilation of visual regularities? Cognitive Science, 42, 692-727. 
Siegelman, N., \& Frost, R. (2015). Statistical learning as an individual ability: Theoretical perspectives and empirical evidence. Journal of Memory and Language, 81, 105-120.

Stanislaw, H., \& Todorov, N. (1999). Calculation of signal detection theory measures. Behavior Research Methods, Instruments, \& Computers, 31(1), 137-149.

Tangen, J. M., Thompson, M. B., \& McCarthy, D. J. (2011). Identifying fingerprint expertise. Psychological Science, 22(8), 995-997.

Thompson, M. B., \& Tangen, J. M. (2014). The nature of expertise in fingerprint matching: experts can do a lot with a little. PLoS ONE, 9(12), 1-23.

Thompson, M. B., Tangen, J. M., \& McCarthy, D. (2013). Expertise in fingerprint identification. Journal of Forensic Sciences, 58(6), 1519-1530.

Towler, A., White, D., \& Kemp, R. I. (2017). Evaluating the feature comparison strategy for forensic face identification. Journal of Experimental Psychology: Applied, 23(1), 47-58.

Turk-Browne, N. B., Jungé, J. A., \& Scholl, B. J. (2005). The automaticity of visual statistical learning. Journal of Experimental Psychology: General, 134(4), 552-564.

Ulery, B. T., Hicklin, R. A., Buscaglia, J., \& Roberts, M. A. (2012). Repeatability and reproducibility of decisions by latent fingerprint examiners. PLoS ONE, 7(3), 1-12.

Vogelsang, M. D., Palmeri, T. J., \& Busey, T. A. (2017). Holistic processing of fingerprints by expert forensic examiners. Cognitive Research: Principles and Implications, 2(1), 15-15.
Von Ahn, L., Maurer, B., McMillen, C., Abraham, D., \& Blum, M. J. S. (2008). recaptcha: Human-based character recognition via web security measures. 321(5895), 1465-1468.

Wickham, H. (2016). ggplot2: elegant graphics for data analysis. United States: Springer.

Wray, J., \& Gates, B. (1996). Problems of recruiting participants for nursing research: a case study. University of Alberta Health Sciences Journal, 1(5), 366-373.

Yin, R. K. (1969). Looking at upside-down faces. Journal of Experimental Psychology, 81(1), 141-145.

Zacks, R. T., \& Hasher, L. (2002). Frequency processing: A twenty-five year perspective. In P. Sedlmeier \& T. Betsch (Eds.), Etc. Frequency Processing and Cognition (pp. 21-36). New York, NY, US: Oxford University Press.

Zhang, H., \& Maloney, L. T. (2012). Ubiquitous log odds: a common representation of probability and frequency distortion in perception, action, and cognition. Frontiers in Neuroscience, 6, 1-14.

Thanks to Wiernik (2019, October 11) for access to the APA preprint template.

Wiernik, B. M. (2019, October 11). Preprint templates. https://doi.org/10.17605/OSF.IO/HSV6A 